\title{
Stability of Anthocyanins in Various Vegetables and Fruits
}

\author{
Kazuya HayASHI, ${ }^{1}$ Naohiro OHARA ${ }^{2}$ and Akio TsukUi ${ }^{3}$ \\ ${ }^{1}$ Wada Sugar Refining Co., Ltd., Department of Development , 3-15-28, Chuo, Edogawa-ku, Tokyo 114, Japan \\ ${ }^{2}$ Department of Agricultural Chemistry, Faculty of Agriculture, Tokyo University of Agriculture, 1-1-1, Sakuragaoka, Setagaya-ku, \\ Tokyo 156, Japan \\ ${ }^{3}$ Tokyo Kasei Gakuin Junior College, 22, Sanbanchou, Chiyoda-ku, Tokyo 102, Japan
}

Received July 13, 1995

\begin{abstract}
Anthocyanins were isolated from 19 kinds of vegetables and fruits. Individual anthocyanins were analyzed by HPLC using a photodiode array detector and were examined for the heating and ultraviolet irradiation stabilities of these anthocyanins. This report deals with the relationship between anthocyanin composition and heating or ultraviolet irradiation stabilities of anthocyanins. Stable anthocyanins showed many anthocyanin peaks and a high ratio of acylated anthocyanin peaks. This result shows that the anthocyanin composition and the rate of acylation affect the stability of anthocyanins.
\end{abstract}

Keywords: various vegetable and fruit anthocyanins, stability, HPLC analysis

Anthocyanins obtained from plants have been used as a coloring material for 'umezuke,' wine, jam and juice, and recently anthocyanins are known to show anti-oxidant activities (Igarashi et al, 1989, 1993; Ishii, 1995).

Several theories have been proposed to explain the stabilization mechanism of anthocyanins such as self-association (Asen et al., 1977), copigmentation (Robinson \& Robinson, 1931) and intramolecular copigmentation (Saito et al., 1972; Goto et al., 1982). Anthocyanins are unstable and easily degraded by heat and light, etc. (Ishii et al., 1990). Therefore, the anthocyanin colors practically change to yellow or fade away during food processing. Studies of various effects on anthocyanin stability in foods, such as heating, light irradiation, $\mathrm{pH}$, metal ions, sugars and organic acids, have been reported (Tsukui, 1988 1989; Tsukui et al., 1989).

In this study, anthocyanins were extracted from 19 kinds of vegetables and fruits, and we examined the relationship between anthocyanin composition, i.e., the number of anthocyanin peaks, and anthocyanin stability to heating and ultraviolet light (UV) irradiation.

\section{Materials and Methods}

Anthocyanin samples Red cabbage (Brassia oleracea L.), perilla 'Katame shiso' (Perilla forma discolor Makino), chicory 'Chicoria rossa' (Cichorium endivia L.), eggplant 'Naga nasu' (Solanum melongena L.), purple onion (Allium cepa L.), red radish (Raphanus sativus L.), red turnip (Brassica campestris L.), black soybean (Glysine max Merr.), water chestnut ( Trapa japonica Flerov), purple yam (Dioscorea alata L.), strawberry 'Nyohou' (Fragaria $\times$ ananassa), red raspberry (Rubus idaeus L.), prune (Prunus domestica L.), grape 'Kaizi' (Vitis vinifera L.), 'Kyohou' (Vitis vinifera L. $\times$ V. labrusca L.), apple 'Sun tsugaru' (Malus pumila Mill.), and sweet potato 'Tanegashima murasaki (TM)' (Ipomoea batatas Lam.) were purchased from the market. Sweet potato varieties 'Yamagawa murasaki (YM)' and 'Kankei 55 (K55)'
(Ipomoea batatas Lam.) were donated by the Sweet Potato Breeding Laboratory of the National Agriculture Research Center.

Red cabbage, eggplant, purple onion, red turnip, and black soybeans were produced in Abiko city of Chiba Prefecture. Perilla was produced in Odawara city of Kanagawa Prefecture. Red radish was produced in Miura city of Kanagawa Prefecture. Water chestnut was produced in the Aso area of Kumamoto Prefecture. Strawberry was produced in the Tochigi area of Tochigi Prefecture. Grape 'Kaizi' and 'Kyohou' were produced in Koufu and Katsunuma cities of Yamanashi Prefecture. Apple was produced in the Tsugaru area of Aomori Prefecture. Sweet potato 'TM' was produced in the Aira area of Kagoshima Prefecture. Sweet potato 'YM' and 'K55' were obtained in the fields of the Tsukuba Sweet Potato Breeding Laboratory. Chicory was imported from Italy. Red raspberry and prune were imported from the USA. Purple yam (powdered) was imported from Philippines.

Preparation of anthocyanins Samples were pulverized with a blender, extracted with $3 \%$ trifluoroacetic acid (TFA) solution overnight at $4^{\circ} \mathrm{C}$ and filtered. The extract was passed through an Amberlite XAD-7 column (30 mm i.d. $\times$ $450 \mathrm{~mm}$ ). The column was washed with $1 l$ of $\mathrm{H}_{2} \mathrm{O}$ and the absorbed anthocyanins were eluted with $0.3 \%$ TFAmethanol. The pigment eluate was concentrated and then dissolved in $0.1 \%$ TFA-methanol, filtered and dried. The residue was dissolved in a small amount of $0.1 \%$ TFAmethanol, and anthocyanin was precipitated by the addition of diethyl ether. The precipitate was collected by centrifugation and dried under vacuum at $40^{\circ} \mathrm{C}$.

The color of $0.2 \mathrm{mg}$ of the anthocyanin dissolved in $1 \mathrm{ml}$ of pH 3.16 Macllvain's buffer was measured by a ZE-2000 colorimeter (Nihon Densyoku, Tokyo) and expressed as on L.a.b. value of the Hunter Solid Diagrams.

HPLC analysis HPLC analysis was carried out with a Waters Millennium ${ }^{\mathrm{TM}}$ 2010J HPLC system, and the absor- 
bance in the range from $220 \mathrm{~nm}$ to $700 \mathrm{~nm}$ was measured with a Waters ${ }^{\text {TM996 }}$ photodiode array detector. Separation was accomplished on a TSK gel ODS-80TM $(4.6 \mathrm{~mm}$ i.d. $\times 25 \mathrm{~cm}$, Tosoh, Tokyo) using an isocratic solution of $\mathrm{CH}_{3} \mathrm{CN}$ and $0.1 \%$ TFA $(1: 4, \mathrm{v} / \mathrm{v})$ at a flow rate of $1.0 \mathrm{ml} / \mathrm{min}$. The anthocyanin composition was determined from the HPLC peak area recorded at $525 \mathrm{~nm}$. Acylated anthocyanin peaks were identified based on absorption at two $\lambda \max$ in $310-335$ nm (Hayashi, 1991).

Heat stability of anthocyanins Anthocyanins dissolved in pH 3.16 Macllvain's buffer and also individual anthocyanin solutions $(0.2 \mathrm{mg} / \mathrm{ml})$ were heated at $80^{\circ} \mathrm{C}$ for $0.0,0.5,1.0,3.0,5.0$ and $18.0 \mathrm{~h}$. After the heat treatment, each anthocyanin solution was cooled in an ice-water bath. The color stabilities of anthocyanins were determined by comparing the pre- and post-heating absorbances at $525 \mathrm{~nm}$.

$U V$ irradiation stability of anthocyanins Anthocyanins dissolved in pH 3.16 Macllvain's buffer and individual anthocyanin solutions $(0.2 \mathrm{mg} / \mathrm{ml})$ were irradiated with ultraviolet light at $20^{\circ} \mathrm{C}$ for $0.0,0.5,1.0,3.0,5.0$ and $18.0 \mathrm{~h}$. The sample solutions were irradiated at a rate of $880 \mu \mathrm{W} / \mathrm{cm}^{2}$ using two $6 \mathrm{~W}$ germicidal lamps $(254 \mathrm{~nm})$. The color stability of each anthocyanin was determined by comparing the preand post-irradiation absorbances at $525 \mathrm{~nm}$.

\section{Results}

Yields and colorimeter readings of anthocyanins from vegetables and fruits The yields of anthocyanins and the colorimeter readings (Hunter solid diagrams) of anthocyanin solutions are shown in Table 1.

The greatest yield (1.30\%) was obtained from grape among 19 kinds of vegetables and fruits. The yields of purple yam, red cabbage, red turnip and strawberry anthocyanins were less than $0.1 \%$ but the yields of anthocyanins from other vegetables and fruits were in the range of $0.15-0.68 \%$.

According to the Hunter solid diagrams, chicory, purple onion, strawberry, raspberry and apple anthocyanins were red with $b$ values of 4.37-13.53 and $a$ values of 21.79-31.29, and red radish and red turnip anthocyanins were yellow-red with $b$ values of $12.20^{-15.06}$ and $a$ values of 39.44-41.53. Red cabbage, perilla, black soybean, water chestnut, sweet potato, 'YM', 'K55', 'TM', purple yam, prune, grape 'Kaizi' and 'Kyohou' anthocyanins were red-purple with $b$ values of -6.64-11.66 and $a$ values of 13.95-58.56. Eggplant anthocyanin was purple with a $b$ value of -9.96 and an $a$ value of 29.31.

Composition of vegetable and fruit anthocyanins The compositions of anthocyanins expressed as the number of anthocyanin peaks by HPLC analysis are shown in Table 2.

Table 2. HPLC analysis of anthocyanin contents of 19 vegetable and fruit anthocyanins.

\begin{tabular}{|c|c|c|c|c|c|c|}
\hline \multirow{2}{*}{ Samples } & \multicolumn{4}{|c|}{ Main peaks $\left.(\%)^{a}\right)$} & \multicolumn{2}{|c|}{ Numbers } \\
\hline & $\mathrm{P}-1$ & $\mathrm{P}-2$ & $\mathrm{P}-3$ & $\mathrm{P}-4$ & Peaks $^{b)}$ & Acylated $^{c)}$ \\
\hline Red cabbage & 38.05 & 20.93 & - & - & 12 & 4 \\
\hline Perilla & 49.92 & 16.72 & - & - & 10 & 3 \\
\hline Chicory & 49.00 & 27.00 & 17.65 & - & 6 & - \\
\hline Eggplant & 93.54 & - & - & - & 4 & 1 \\
\hline Purple onion & 41.51 & 10.34 & 10.24 & - & 10 & 4 \\
\hline Red radish & 39.07 & 33.20 & 10.78 & - & 8 & 3 \\
\hline Red turnip & 63.16 & 12.10 & - & - & 5 & 4 \\
\hline Black soybean & 71.40 & 14.81 & - & - & 6 & 1 \\
\hline Water chestnut & 84.62 & - & - & - & 5 & - \\
\hline Yamaga murasaki & 29.97 & 15.68 & 14.60 & 14.51 & 9 & 8 \\
\hline Kankei 55 & 26.94 & 16.45 & 12.14 & 10.02 & 11 & 6 \\
\hline$\underset{\text { murasaki }}{\text { Tanegashima }}$ & 24.67 & 23.56 & - & - & 13 & 6 \\
\hline Purple yam & 37.96 & 25.75 & 11.00 & 10.78 & 9 & 4 \\
\hline Strawberry & 90.96 & - & - & - & 3 & - \\
\hline Raspberry & 62.05 & 21.22 & - & - & 6 & - \\
\hline Kyohou & 49.69 & 21.15 & 10.88 & - & 7 & 2 \\
\hline Kaizi & 74.63 & 12.93 & 10.17 & - & 3 & - \\
\hline Prune & 44.24 & 40.22 & - & - & 6 & - \\
\hline Apple & 84.48 & - & - & - & 3 & - \\
\hline
\end{tabular}

a) Anthocyanins contain over $10 \%,{ }^{\text {b) }}$ anthocyanin peak numbers of contents over $1 \%,{ }^{c}$ number of acylated anthocyanin peaks.
Table 1. Anthocyanin yields and $L, a, b$, values of 19 vegetables and fruits

\begin{tabular}{lcrrr}
\hline \multirow{2}{*}{ Samples } & Yield & \multicolumn{3}{c}{ Hunter's chromatic value } \\
\cline { 4 - 5 } & $(\boldsymbol{\%})$ & $L$ & $a$ & \multicolumn{1}{c}{$b$} \\
\hline Red cabbage & 0.07 & 65.51 & 33.24 & -6.64 \\
Perilla & 0.42 & 68.97 & 31.47 & -5.41 \\
Chicory & 0.22 & 71.23 & 39.29 & 5.01 \\
Eggplant (skin) & 0.49 & 82.02 & 29.31 & -9.96 \\
Purple onion & 0.15 & 79.46 & 33.29 & 2.10 \\
Red radish (skin) & 0.43 & 76.95 & 41.53 & 12.20 \\
Red turnip & 0.08 & 77.51 & 39.44 & 15.06 \\
Black soybean & 0.45 & 70.74 & 23.82 & 11.66 \\
Water chestnut & 0.12 & 91.12 & 13.95 & 0.94 \\
Sweet potato Yamagawa murasaki & 0.68 & 66.67 & 31.02 & -8.26 \\
Sweet potato Kankei 55 & 0.38 & 60.32 & 35.53 & -7.05 \\
Sweet potato Tanegashima murasaki & 0.18 & 71.11 & 25.17 & -5.69 \\
Purple yam & 0.05 & 58.74 & 58.56 & 3.19 \\
Strawberry & 0.09 & 90.11 & 13.52 & 13.53 \\
Raspberry & 0.28 & 75.53 & 21.79 & 4.37 \\
Prune (skin) & 0.36 & 71.45 & 15.35 & -0.77 \\
Grape Kyohou (skin) & 1.30 & 71.54 & 35.63 & 11.21 \\
Grape Kaizi (skin) & 0.15 & 77.28 & 19.42 & 9.68 \\
Apple (skin) & 0.22 & 86.88 & 22.60 & 4.90 \\
\hline & & & &
\end{tabular}

Table 3. Effect of heating on the stability of 19 vegetable and fruit anthocyanins.

\begin{tabular}{lcccccc}
\hline \multirow{2}{*}{ Anthocyanins } & \multicolumn{6}{c}{ Relative absorbance $(525 \mathrm{~nm})$} \\
\cline { 2 - 7 } & 0.0 & 0.5 & 1.0 & 3.0 & 5.0 & $18.0(\mathrm{~h})$ \\
\hline Red cabbage & 100 & 79 & 77 & 74 & 73 & 60 \\
Perilla & 100 & 76 & 74 & 69 & 64 & 44 \\
Chicory & 100 & 87 & 80 & 72 & 65 & 31 \\
Eggplant & 100 & 72 & 66 & 56 & 48 & 22 \\
Purple onion & 100 & 89 & 84 & 74 & 68 & 38 \\
Red radish & 100 & 81 & 81 & 76 & 71 & 54 \\
Red turnip & 100 & 80 & 80 & 74 & 69 & 46 \\
Black soybean & 100 & 63 & 60 & 51 & 44 & 19 \\
Water chestnut & 100 & 87 & 85 & 75 & 65 & 40 \\
Yamagawa murasaki & 100 & 89 & 89 & 86 & 84 & 71 \\
Kankei 55 & 100 & 95 & 94 & 92 & 89 & 75 \\
Tanegashima murasaki i & 100 & 80 & 79 & 78 & 76 & 63 \\
Purple yam & 100 & 97 & 97 & 94 & 92 & 77 \\
Strawberry & 100 & 86 & 86 & 76 & 69 & 24 \\
Raspberry & 100 & 93 & 86 & 76 & 67 & 30 \\
Kyohou & 100 & 75 & 70 & 65 & 58 & 32 \\
Kaizi & 100 & 58 & 57 & 49 & 42 & 17 \\
Prune & 100 & 80 & 78 & 70 & 65 & 33 \\
Apple & 100 & 89 & 80 & 60 & 47 & 12 \\
\hline
\end{tabular}


Table 4. Effect of UV irradiation on the stability of 19 vegetable and fruit anthocyanins.

\begin{tabular}{lrrrrrrrr}
\hline & \multicolumn{7}{c}{ Relative absorbance $(525 \mathrm{~nm})$} \\
\cline { 2 - 9 } Anthocyanins & 0.0 & 0.5 & 1.0 & 2.0 & 3.0 & 4.0 & 5.0 & $\begin{array}{c}18.0 \\
\text { (h) }\end{array}$ \\
\hline Red cabbage & 100 & 99 & 97 & 91 & 83 & 74 & 74 & 43 \\
Perilla & 100 & 99 & 97 & 89 & 86 & 78 & 78 & 48 \\
Chicory & 100 & 88 & 86 & 71 & 64 & 59 & 56 & 25 \\
Eggplant & 100 & 93 & 80 & 64 & 60 & 42 & 36 & 18 \\
Purple onion & 100 & 92 & 87 & 79 & 74 & 71 & 64 & 47 \\
Red radish & 100 & 107 & 109 & 110 & 107 & 105 & 102 & 69 \\
Red turnip & 100 & 105 & 109 & 111 & 109 & 107 & 104 & 59 \\
Black soybean & 100 & 94 & 90 & 85 & 76 & 67 & 58 & 26 \\
Water chestnut & 100 & 98 & 98 & 95 & 93 & 92 & 92 & 60 \\
Yamagawa murasaki & 100 & 100 & 99 & 98 & 96 & 92 & 93 & 74 \\
Kankei 55 & 100 & 100 & 99 & 99 & 99 & 97 & 95 & 80 \\
Tanegashima murasaki & 100 & 97 & 96 & 94 & 92 & 90 & 87 & 68 \\
Purple yam & 100 & 98 & 96 & 94 & 92 & 90 & 89 & 65 \\
Strawberry & 100 & 102 & 65 & 78 & 62 & 50 & 41 & 6 \\
Raspberry & 100 & 94 & 88 & 76 & 66 & 56 & 48 & 8 \\
Kyohou & 100 & 98 & 95 & 89 & 83 & 76 & 68 & 44 \\
Kaizi & 100 & 106 & 101 & 85 & 69 & 60 & 55 & 22 \\
Prune & 100 & 94 & 88 & 78 & 69 & 63 & 58 & 18 \\
Apple & 100 & 100 & 97 & 87 & 77 & 66 & 58 & 10 \\
\hline
\end{tabular}

HPLC analysis revealed that red cabbage, perilla, purple yam, red radish, purple onion and sweet potato anthocyanins were separated into 8-13 pigment peaks. Other vegetable and fruit anthocyanins had less than 7 anthocyanin peaks.

Heat stability of vegetable and fruit anthocyanins Anthocyanins stable to heating at $80^{\circ} \mathrm{C}$ for $18 \mathrm{~h}$ were purple yam anthocyanins (the percentage residual being $77 \%$ ), sweet potato 'K55' (75\%), 'YM' (71\%), 'TM' (63\%) and red cabbage (60\%) (Table 3). By HPLC analysis, these anthocyanins had many peaks including acylated anthocyanin peaks. However, anthocyanins composed of a small number of anthocyanin peaks were unstable as shown for eggplant, chicory, strawberry, raspberry and apple (Table 3 ).

$U V$ irradiation stability of vegetable and fruit anthocyanins The results of UV irradiation for $18 \mathrm{~h}$ are shown in Table 4. The anthocyanins stable to UV irradiation were sweet potato ' $\mathrm{K} 55$ ' (the residual color ratio being $80 \%$ ), 'YM' (74\%), red radish $(69 \%)$ and 'TM' $(68 \%)$. These anthocyanins had a high ratio of acylated anthocyanin peaks. However, anthocyanins with low acylated ratios such as grape, strawberry, raspberry and apple were unstable. Red radish and red turnip were extremely stable and even increased slightly in absorbance at $525 \mathrm{~nm}$ in the first $5 \mathrm{~h}$.

Relationship between residual color ratio and anthocyanin peaks The relationships between color stability of anthocyanins and anthocyanin composition are shown in Fig. 1.

Upon heating, a positive correlation was observed between the residual color ratios and the number of anthocyanin peaks $(r=0.79, p<0.01)$. A positive correlation also existed between the residual color ratios and the number of acylated anthocyanin peaks $(r=0.85, p<0.01)$.

Concerning the effects of UV irradiation, a positive correlation was observed between the residual color ratios and the number of acylated anthocyanin peaks $(r=0.83, p<$ 0.01 ).

\section{Discussion}

Generally, anthocyanins become stable due to trance formation of the flavylium cation structure in acidic aqueous solution.

Anthocyanins are very unstable in weakly acidic or neutral aqueous solution, and these colors rapidly disappear under such conditions. Anthocyanin discoloring is generally recognized to be caused by transforming of the anhydro base formation which is easily hydrated into the colorless pseudobase formation.

Keeping the colors is based on preventing the hydration of anthocyanins. Color stabilization mechanisms of anthocyanin have already been proposed such as metal ion complexation (Hayashi et al., 1958), complex formation of anthocyanins with flavonoid copigments (co-pigmentation) (Robinson \& Robinson, 1931), self-association (Asen et al., 1977; Hoshino et al., 1982) and intramolecular sandwich-type stacking of the anthocyanin nucleus and acyl groups (intramolecular copigmentation) (Saito et al., 1972; Goto et al, 1982; Goto \& Kondo, 1988).

Tsukui $(1988,1989)$ and Tsukui et al. (1989) reported that various factors affect anthocyanin stability in foods, such as heating, light irradiation, $\mathrm{pH}$, metal ions, sugars and organic acids.
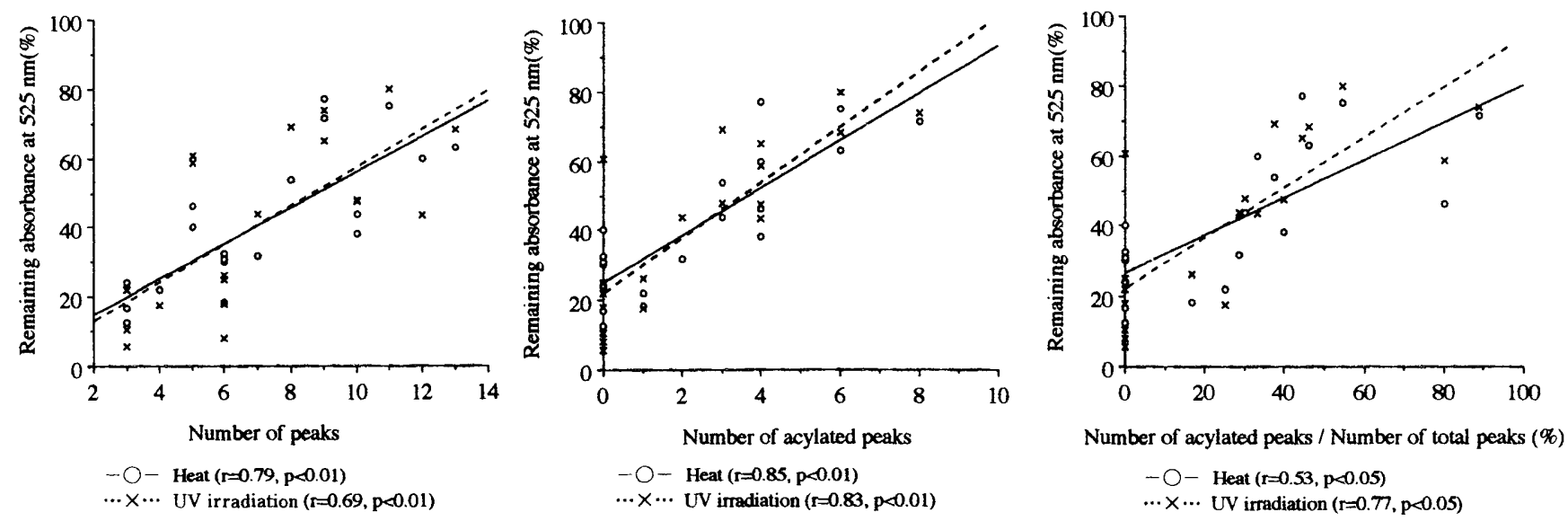

Fig. 1. Relationships between anthocyanin stabilities and anthocyanin composition 
In this study, we extracted and purified anthocyanins from 19 kinds of vegetables and fruits and examined the relationship between anthocyanin composition and the stability of anthocyanins.

A positive correlation was observed between the color stability on heating and the number of anthocyanin peaks and also acylated anthocyanins. Anthocyanins composed of many anthocyanins and acylated anthocyanins, such as purple yam, sweet potato and red cabbage, were stable. This would indicate that anthocyanin stability is caused by self-association.

The stability to UV irradiation was different from the stability to heating. A positive correlation was observed between residual color ratios and the number of acylated anthocyanin peaks. Anthocyanins of sweet potato were the most stable, which were acylated with many organic acids such as caffeic acid, ferulic acid and $\rho$-hydroxybenzoic acid (Shimizu \& Nakamura, 1994; Miyazaki et al, 1991). The number of acylated anthocyanins was more influential on the UV irradiation stability of anthocyanins than the number of anthocyanins. From this result, it was presumed that the UV irradiation stability of anthocyanin was derived from intramolecular sandwich-type stacking with acyl groups. However, red radish and red turnip were extremely stable. In this case, anthocyanin stability cannot be elucidated by only self-association or intramolecular copigmention. These results are interesting and worthy of further study.

Acknowledgments We thank Dr. K. Komaki of the Sweet Potato Breeding Lab., Nat. Agr. Res. Center, MAFF, Japan, for his gift of sweet potato varieties 'Yamagawa Murasaki' and 'Kankei 55'.

\section{References}

Asen, S., Stewart, R.N. and Norris, K.H. (1977). Co-pigmentation of anthocyanins in plant tissues and its effect on color. Phytochemistry, 16, 1118-1119.

Goto, T., Kondo, T., Tamura, H., Imagawa, H., Iino, A. and Takeda, K. (1982). Structure of gentiodelphin, an acylated anthocyanin isolated from Gentiana makinoi, that is stable in dilute aqueous solution. Tetrahedron Lett., 23, 3695-3698.
Goto, T. and Kondo, T. (1988). Flower colors-Structure, stability, and intramolecular stacking of highly acylated anthocyanins. Yukigouseikagaku, 46, 426-435 (in Japanese).

Hayashi, K., Abe, Y. and Mitsui, S. (1958). Blue anthocyanin from the flowers of commelina, the crystallisation and some properties there of studies on anthocyanins XTT. Proc. Jpn. Acad., 34, 373-378.

Hayashi, K. (1991). Qualitative analysis of pigments. In "Plant Pigment" ed. by Hayashi, K. Yokendo, Tokyo, pp. 169-171 (in Japanese).

Hoshino, T., Matsumoto, U., Goto, T. and Harada, N. (1982). Evidence for the self-association of anthocyanins Iv. PMR spectroscopic evidence for the vertical stacking of anthocyanin molecules. Tetrahedron Lett., 23, 433-436.

Igarashi, K., Takanashi, K., Makino, M. and Yasui, T. (1989) Antioxidative activity of major anthocyanin isolated from wild grapes. Nippon Shokuhin Kogyo Gakkaishi, 36, 852-856.

Igarashi, K., Yoshida, T. and Suzuki, E. (1993). Antioxidative activity of Nasunin in Chouja-Nasu. Nippon Shokuhin Kogyo Gakkaishi, 40, 138-143.

Ishii, A. (1995). Antioxidative activity of colorful potato anthocyanin Seibutu to Kagaku, 33, 148-150 (in Japanese).

Ishii, C., Suzuki, A., Kurata, M. and Omote, Y. (1990). Thermal stability of anthocyanins from eggplant, Solanum melongena $\mathrm{L}$. Nippon Shokuhin Kogyo Gakkaishi, 37, 984-987 (in Japanese).

Miyazaki, T., Tsuzuki, W. and Suzuki, T. (1991). Composition and structure of anthocyanins in the periderm and flesh of sweet potatoes. Engeigaku Gakkaishi, 60, 217-224 (in Japanese).

Robinson, G.M. and Robinson, R. (1931). A survey of anthocyanins. I. Biochem. J., 25, 1687-1705.

Saito, N., Osawa, Y. and Hayashi, K. (1972). Isolation of a blue-violet pigment from the flower of Platycodon grandiflorum. Phytochemistry, 85, 105-110

Shimizu, T. and Nakamura, M. (1994). Anthocyanins. In "Syokuyou tennen shikiso" ed. by Fuzii, M. Kohrin, Tokyo, pp. 71-104 (in Japanese).

Tsukui, A. (1988). Stability of anthocyanin pigments of Philippine powdered purple yam - Effect of $\mathrm{pH}$, light and oxygen - Nippon Kasei Gakkaishi, 39, 209-215 (in Japanese).

Tsukui, A. (1989). Stability of anthocyanin pigments of Philippine powdered purple yam -Effect of inorganic salts, organic acids, sugars, phenols, L-ascorbic acids and hydrogen peroxide - . Nippon Kasei Gakkaishi, 40, 15-22 (in Japanese).

Tsukui, A., Kobayashi, K. and Saito, N. (1989). Stability of anthocyanin pigments of Philippine powdered purple yam-On the comparison of powdered purple yam and papilionanceae- Nippon Kasei Gakkaishi, 40, 115-119 (in Japanese). 